\title{
Ischemic Brain Injury Secondary to Severe Systemic Loxoscelism
}

\author{
Matthew P Soape MD, Deephak Swaminath MD, Mark Whealy MD, Vipul Desai MD
}

\begin{abstract}
Systemic loxoscelism is a rare complication from the bite of spiders in the genus Loxosceles. These bites usually cause painful indurated skin reactions, including necrosis, and occasionally cause systemic complications, such as rhabdomyolysis, acute renal failure, and disseminated intravascular coagulation. Our case had multiple systemic complications, including bilateral globus pallidus infarcts with right arm weakness.
\end{abstract}

Key words: spider bite, Loxosceles reclusa, systemic loxoscelism

\section{INTRODUCTION}

Rhabdomyolysis, acute renal failure, and disseminated intravascular coagulation (DIC) frequently occurs in patients with severe systemic loxoscelism. The case presented here had these complications plus acute respiratory failure and bilateral globus pallidus lesions. A literature search failed to recover any cases that reported central nervous system (CNS) lesions associated with systemic loxoscelism. In this article, we discuss the pathophysiology underlying systemic loxoscelism that probably explains the ischemic injury to the CNS.

\section{Case Presenttion}

A 47-year-old Caucasian man was found unresponsive in Carlsbad, NM, was unable to protect his airways, and was intubated at the scene. In the $\mathrm{ER}$, the patient had a temperature of $100.9^{\circ} \mathrm{F}$, a BP of $173 / 104 \mathrm{mmHg}$, and a pulse rate of 117 beats per

Corresponding author: Matthew Soape MD

Contact Information: matthew.soape@ttuhsc.edu

DOI: 10.12746/swrccc2014.0206.076 minute. He had swelling and erythema on the parieto-occipital aspect of his head. He had diffuse crackles in both lungs. His CBC included WBC $9.4 \mathrm{k} / \mu \mathrm{L}$, $\mathrm{Hb} 15.7 \mathrm{gm} / \mathrm{dl}$, Hct $45.2 \%$, and platelet $92 \mathrm{k} / \mu \mathrm{L}$. His labs showed evidence of rhabdomyolysis (CK 57,125 IU/L), acute renal failure (BUN $37 \mathrm{mg} / \mathrm{dL}$; $\mathrm{Cr} 4.1 \mathrm{mg} /$ $\mathrm{dL}$ ), hepatitis (ALT 5,721 intl units/L; AST 12,831 intl units/L), and coagulopathy (PT $20.9 \mathrm{sec}$; INR 2.02; PTT $35.5 \mathrm{sec}$, D-Dimer 11,313 ng/ml). He also had a high LDH (5,372 units/L) and developed a mild anemia during his hospitalization $(\mathrm{Hb} 15.7 \mathrm{gm} / \mathrm{dl}$ on admission with a decrease to $11.8 \mathrm{gm} / \mathrm{dl}$ on hospital day six associated with an increase in bilirubin from 0.8 $\mathrm{mg} / \mathrm{dl}$ on admission to $1.3 \mathrm{mg} / \mathrm{dl}$ day four). During the first 24 hours of admission, a necrotic lesion formed at the site of the swelling on his head (Figure 1). This lesion had a raised edge with surrounding erythema and grew to a circumference of $5 \mathrm{~cm}$. The dermatology consult concluded the lesion was most likely secondary to a spider bite. No information regarding any spider bite or sighting could be obtained. The patient was successfully extubated and transferred to floor status after day three. 


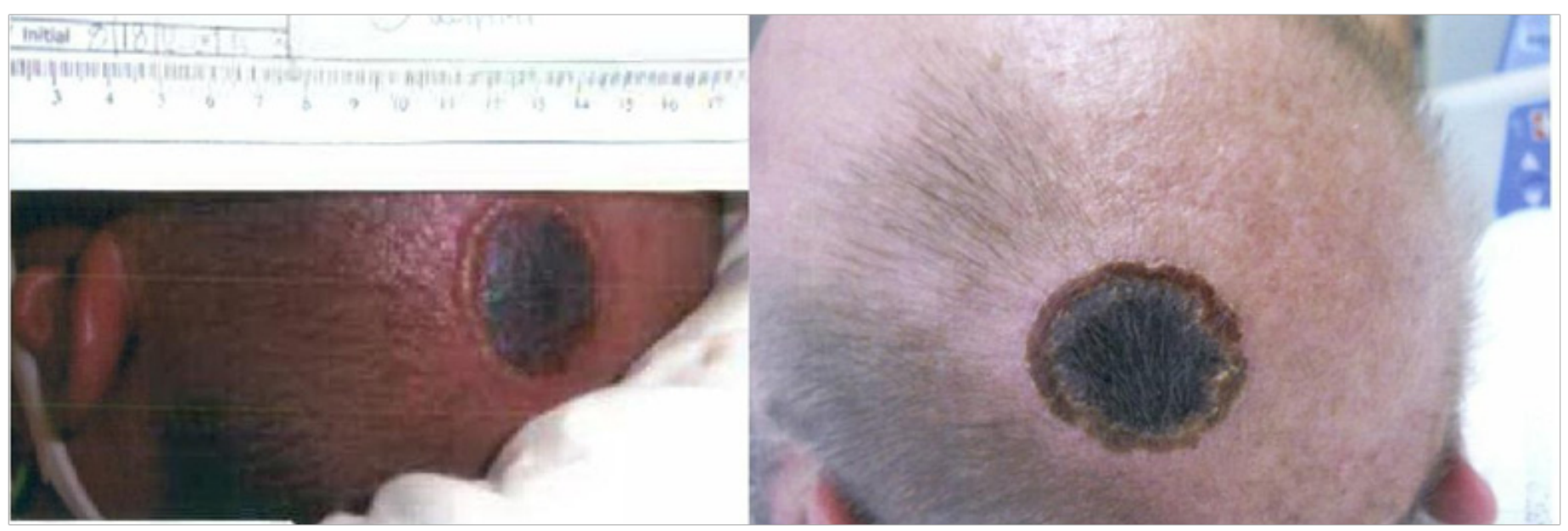

Figure 1: Photographs of the site of suspected brown recluse envenomation on the second day of admission (left) and 10 days later (right).

On day five, he noticed weakness of his right upper extremity (RUE). A CT scan of his head showed a subacute infarct in the internal capsule, and a subsequent MRI demonstrated infarcts in both globus pallidi (Figure 2). The patient continued to spike fevers throughout his hospitalization. On day 10 of hospitalization, the patient had a large indurated area on his right lateral thigh. An Infectious Disease consult recommended starting vancomycin for cellulitis. All of his labs and symptoms, including his RUE weakness, improved throughout his remaining hospital stay. He was discharged home on day 12 of hospitalization with instructions to continue vancomycin for two weeks.

\section{Discussion}

Systemic loxoscelism is a rare complication from the bite from spiders in the genus Loxosceles. These bites usually cause painful indurated skin reactions but can cause necrosis and ulcers. Necrotic ulcers may take months to heal and require debridement and skin grafting. Some patients develop systemic signs and symptoms, including mild fever, myalgias, arthralgias, rhabdomyolysis, hemolytic anemia,

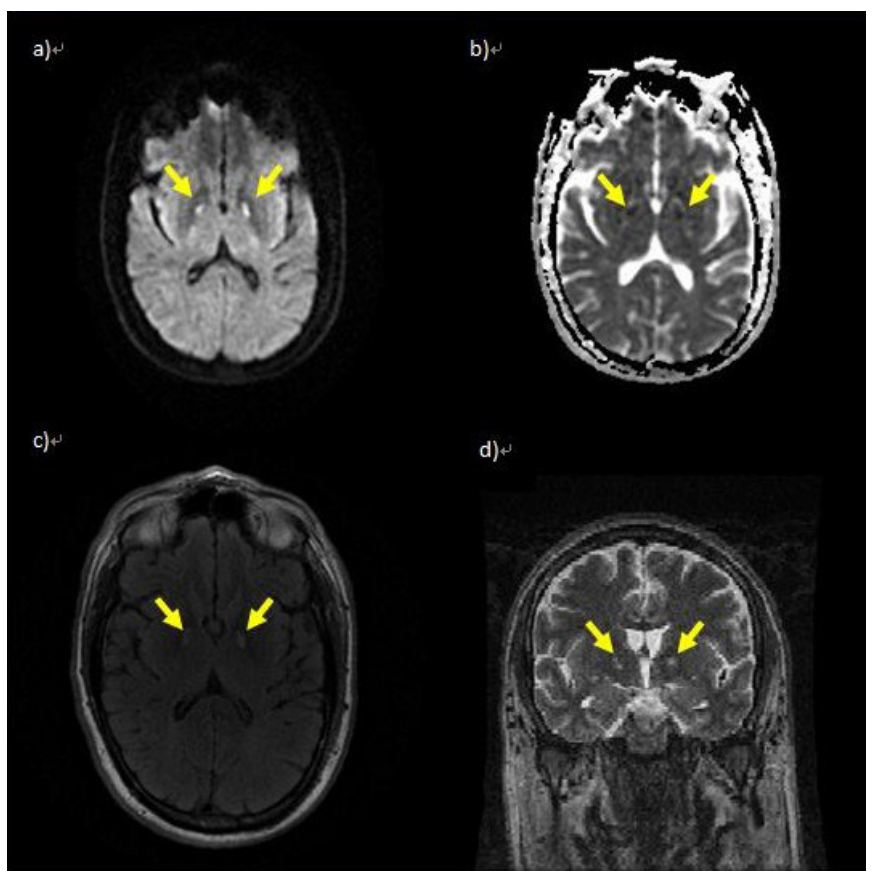

Figure 2: MRI head of our patient on day four of admission after complaining of right upper extremity weakness. a) Diffusion weighted imaging showing bilateral globus pallidus hyperintensities which correlate with hypointensities seen on the b) diffusion coefficient image c) T2 FLAIR d) coronal T2 images. Yellow arrows pointing to bilateral globus pallidus hyperintesities. 
DIC, and acute renal failure, and have very complicated hospital courses. ${ }^{1}$ The brown recluse spider (Loxosceles reclusa) is frequently responsible for these bites in the southern United States.

This patient presented with erythematous swelling on his scalp that progressed to a large area of necrosis consistent with a brown recluse spider bite. Although we cannot be certain that this was a spider bite, it is strongly suspected based on the available information, the clinical course, and the fact that the brown recluse is endemic to the area where the incident occurred. The lack of confirmation is not unusual, and in the nine case reports with 16 patients there were only two confirmed brown recluse spider bites (the spider was captured and brought in for identification) and two sightings. ${ }^{2-10}$ None of these cases had CNS involvement. The most common severe reactions to a Loxosceles bite in these case reports were hemolysis, rhabdomyolysis, acute kidney injury, and death. Since this diagnosis is often one of exclusion, a thorough differential diagnoses is always needed. Other causes for rhabdomyolysis, including recreational drug use and statin therapy, seemed unlikely. The patient had a negative urine drug screen and had only started statin therapy a few days prior to hospitalization. He had no history of anemia and hereditary causes of hemolysis were unlikely given his age. Sepsis could explain his DIC, but blood cultures taken on admission were negative at the time when the coagulopathy and thrombocytopenia were at their worst lab values. A separate blood culture on day five grew Staphylococcus epidermidis, a common skin bacterium which could have been introduced secondary to his spider bite. Also DIC occurs more frequently in Gram negative sepsis than Gram positive sepsis. Cancer and trauma were also ruled out.

This case demonstrates the possibility of CNS injury can occur in systemic loxoscelism. This patient's infarcts could reflect hypoxia from the acute respiratory failure or a direct effect of venom toxins on the CNS circulation. If the CNS infarcts were secondary to hypoxia, the CNS involvement should have been more diffuse with laminar cortical necrosis and not just localized to the globus pallidus. Carbon monoxide poisoning can cause diffuse cerebral injury, but his daughter who was sleeping in the same room had no adverse effects. The ischemic injury was probably secondary to microvascular thrombosis secondary to DIC. A possible mechanism is the induction of a hypercoagulable state by the $35 \mathrm{kD}$ sphingomyelinase $\mathrm{D}$ in Loxosceles venom. This protein cleaves thrombomodulin and endothelial protein $\mathrm{C}$ receptor through the activation of an endogenous metalloproteinase. These cells can not normally express surface proteins to aid in the thrombin induced activation of protein $\mathrm{C}$, and this process could cause DIC and thrombosis."

Also, Loxosceles venom has direct cytotoxic effects on the endothelial cells which produce morphological changes and detachment from the subendothelial layer. ${ }^{12}$ This would expose the subendothelial collagen leading to binding of von Willebrand factor and subsequent platelet activation and thrombosis. Consequently, the combination of vascular injury and DIC with fibrin deposition causes tissue ischemia and injury. The localization to the globus pallidi is unexplained.

In conclusion, this case has a unique presentation of systemic loxoscelism. He had common features, such as hemolysis, acute kidney injury, and rhabdomyolysis, and unique infarcts in the basal ganglia. This finding has not been reported in the literature to date.

Author Affiliation: Matthew P Soape and Deephak
Swaminath are residents in Internal Medicine at TTUHSC
in Lubbock, TX. Vipul Desai is an infectious disease faculty
member in the Department of Internal Medicine at TTUHSC
in Lubbock, TX. Mark Whealy is in the Department of
Neurology at the Mayo Clinic in Rochester, MN.

Received: $02 / 03 / 2014$

Accepted: 04/11/2014

Reviewers: Vaqur Ahmed MD

Published electronically: 04/15/2014

Conflict of Interest Disclosures: none 


\section{REFERENCES}

1. Malaque $\mathrm{C} \mathrm{M}$, et al. Clinical Picture and Laboratorial Evaluation in Human Loxoscelism. Toxicon. 2011; 58(8): 664-71.

2. de Souza A L, et al. Loxosceles Venom-Induced Cytokine Activation, Hemolysis, and Acute Kidney Injury. Toxicon. 2008; 51(1): 151-6.

3. Elbahlawan L M, et al. Severe Systemic Reaction to Loxosceles Reclusa Spider Bites in a Pediatric Population. Ped Emerg Care. 2005; 21(3): 177-80.

4. Franca F O, Barbaro KC, Abdulkader RC. Rhabdomyolysis in Presumed Viscero-Cutaneous Loxoscelism: Report of Two Cases. Transactions of the Royal Society of Tropical Medicine \& Hygiene. 2002; 96(3): 287-90.

5. Goto C S, Abramo TJ, Ginsburg CM. Upper Airway Obstruction Caused by Brown Recluse Spider Envenomization of the Neck. Am J Emerg Med. 1996; 14(7): 660-2.

6. Leung LK, Davis R. Life-Threatening Hemolysis Following a Brown Recluse Spider Bite. J Tenn Med Assoc. 1995; 88(10): 396-7.

7. Murray LM, Seger DL. Hemolytic Anemia Following a Presumptive Brown Recluse Spider Bite. J Tox. Clin Tox. 1994; 32(4): 451-6.

8. Rosen J L, et al. Emergency Department Death from Systemic Loxoscelism. Annu Emerg Med. 2012; 60(4): 439-41.

9. Taylor EH, Denny WF. Hemolysis, Renal Failure and Death, Presumed Secondary to Bite of Brown Recluse Spider. Southern Med J. 1966; 59(10): 1209-11.

10. Williams ST, et al. Severe Intravascular Hemolysis Associated with Brown Recluse Spider Envenomization. A Report of Two Cases and Review of the Literature. Am J Clin Path. 1995; 104(4): 463-7.

11. van den Berg CW, et al. Loxosceles Spider Venom Induces the Release of Thrombomodulin and Endothelial Protein C Receptor: Implications for the Pathogenesis of Intravascular Coagulation as Observed in Loxoscelism. J Thrombosis and Haemostasis. 2007; 5(5): 989-95.

12. Paludo KS, et al. The Effect of Brown Spider Venom on Endothelial Cell Morphology and Adhesive Structures. Toxicon. 2006; 47(8): 844-53. 